\title{
A voxel based morphometry study on mild cognitive impairment
}

\author{
C Pennanen, C Testa, M P Laakso, M Hallikainen, E-L Helkala, T Hänninen, M Kivipelto, \\ M Könönen, A Nissinen, S Tervo, M Vanhanen, R Vanninen, G B Frisoni, H Soininen
}

J Neurol Neurosurg Psychiatry 2005;76:1 1-14. doi: 10.1136/jnnp.2004.035600

See end of article for authors' affiliations ......................

Correspondence to: Dr H Soininen, Department of Neurology, Bldg. 5, Kuopio University Hospital, POB 1777, 70211

Kuopio, Finland;

hilkka.soininen@kuh.fi

Received 2 January 2004

In revised form

22 March 2004

Accepted 19 April 2004
Background: Mild cognitive impairment $(\mathrm{MCl})$ is the most widely used concept in classifying cognitive impairment in the elderly who do not fulfil the criteria for dementia. $\mathrm{MCl}$ is considered to confer an increased risk of progressing to dementia and most often Alzheimer's disease (AD). Various approaches such as imaging of the brain have been applied to predict the conversion of $\mathrm{MCl}$ to dementia. A number of volumetric magnetic resonance imaging (MRI) studies have detected atrophy of the medial temporal lobe in subjects with $\mathrm{MCl}$, but for the other cerebral regions the results have been inconsistent.

Objective: To study the pattern of brain atrophy in $\mathrm{MCl}$.

Methods: Thirty two controls and 51 individuals with $\mathrm{MCl}$ deriving from population based cohorts were studied by MRI using voxel based morphometry. The threshold of $t$ maps was set at $p<0.001$.

Results: Individuals with $\mathrm{MCl}$ had significant unilateral atrophy in the medial temporal lobe on the right side. Less extensive atrophy was found elsewhere-for example, in the temporal lobe, left superior parietal lobule, left anterior cingulate gyrus, and bilaterally in the thalami.

Conclusions: The MRI findings in $\mathrm{MCl}$ resemble those seen in early $A D$.
M ild cognitive impairment (MCI) is currently the most widely used concept in classifying the cognitive impairment in the elderly who do not fulfil the criteria for dementia. This is important and challenging from at least two points of view. Firstly, in 2001 the Report of the Quality Standards Subcommittee of the American Academy of Neurology reviewed a number of studies and, even though these studies had used various criteria for MCI, they pointed to an annual conversion rate of $6 \%-25 \%$ from MCI to Alzheimer's disease $(\mathrm{AD})$, a rate greatly exceeding that seen in the normal age matched population. ${ }^{1}$ Thus, investigating MCI might provide a means to study $\mathrm{AD}$ in its earliest phases. Secondly, if MCI were indeed to be a significant predictor of future $\mathrm{AD}$, this might prove beneficial in terms of preventive or interventive measures for $\mathrm{AD}$. However, some recent longitudinal population based studies have cast some doubt on the concept of MCI. Ritchie et al suggested that MCI is a poor predictor of dementia; that the group is unstable, with cases changing category almost yearly, and called for modifications to the current criteria. ${ }^{2}$ In the study by Larrieu et al, an annual conversion rate of $8.3 \%$ was observed during a five year period, but again the cases had a tendency to fluctuate, and far more than $40 \%$ of MCI cases reverted to normal instead of progressing to dementia during follow up. ${ }^{3}$

One way of addressing the issue could be to use imaging studies, which do seem to have some potential to detect early $\mathrm{AD}$. Indeed, a number of volumetric magnetic resonance imaging (MRI) studies have detected temporal atrophy (the most common MRI finding in AD) to some degree in cases with MCI as well; however, for the other cerebral regions the results have been rather inconsistent. ${ }^{45}$ In addition to the traditional volumetric methods, recent advances in image analysis have produced new tools to map the entire brain instead of being restricted to single regions. These techniques can be done with relatively little individual effort and have high reproducibility. ${ }^{6-8}$ Perhaps the most widely used of these is voxel based morphometry (VBM), which has not been applied to MCI, with one exception ${ }^{9}$ where 22 patients with amnestic MCI were compared with 22 healthy controls and found to have significant grey matter loss predominantly affecting the hippocampal region and anterior cingulate gyri, and extending into the temporal neocortex. Therefore, the aim of our study was to try to replicate the findings of the preliminary study of MCI in a larger sample, by using more recent techniques related to VBM, and by examining a group of cases with MCI who fulfil probably the most dominant criteria (the first version of the Mayo criteria) for MCI.

\section{MATERIALS AND METHODS}

\section{Participants}

The study included 51 individuals with MCI and 32 controls, derived from two population based cohorts in which cognitive functions of the elderly had been evaluated in the Department of Neurology, Kuopio University Hospital, Kuopio, Finland..$^{10}{ }^{11}$ In both cohorts, the evaluation consisted of a structured interview including clinical dementia rating (CDR) scale and an extensive neuropsychological assessment described in detail elsewhere. ${ }^{12}$ The scoring of the CDR was independent of the scores obtained from neuropsychological tests.

The controls showed no impairment in the cognitive tests, and had no history of neurological or psychiatric diseases. MCI was diagnosed using the criteria proposed by Mayo Clinic Alzheimer's Disease Research Center. Recently, these criteria have been modified, but when our study was conducted the criteria required: (1) memory complaint by patient, family, or physician; (2) normal activities of daily living; (3) normal global cognitive function; (4) objective impairment in memory or in one other area of cognitive function as evident by scores $>1.5$ SD below the age appropriate mean; (5) CDR score of 0.5, and (6) absence of

Abbreviations: $A D$, Alzheimer's disease; $C D R$, clinical dementia rating; CSF, cerebrospinal fluid; GM, grey matter; HERA, hemispheric encoding/retrieval asymmetry; $M C l$, mild cognitive impairment; MRI, magnetic resonance imaging; VBM, voxel based morphometry; WM, white matter. 
dementia. ${ }^{13}{ }^{14}$ The ApoE genotype was determined from blood leukocytes as described previously. ${ }^{15}$

The study was approved by the local ethics committee, and all the participants gave informed consent for their participation in the study.

\section{Magnetic resonance imaging}

Patients and controls underwent a high resolution MRI at the Kuopio University Hospital, Kuopio, Finland. The MR images were acquired using a 1.5 Tesla Vision Magnetom scanner (Siemens, Erlangen, Germany), with a 3D gradient echo technique $\left(\mathrm{TR}=9.7 \mathrm{~ms}, \mathrm{TE}=4 \mathrm{~ms}\right.$, flip angle $=10^{\circ}$, field of view $=250 \mathrm{~mm}$, and the acquisition matrix was $256 \times 256$ resulting in $1282.0 \mathrm{~mm}$ thick Tl weighted coronal slices). T2 weighted images were also acquired, and used to study the possibility of vascular pathology in the cases. None of the subjects was excluded from the study on the basis of T2 weighted images. To control for differences in head size, intracranial area measured at the level of the anterior commissure was used. ${ }^{12}$

\section{Voxel based morphometry preprocessing}

After removing the voxels below the cerebellum with MRIcro software (www.psychology.nottingham.ac.uk/staff/crl/mricro. html) ${ }^{16}$ the MR images were analysed with SPM99 (www. fil.ion.ucl.ac.uk/spm) running under Matlab 5.3 (Mathworks, Sherborn, MA, USA).

The MR images were preprocessed following a protocol which includes (1) generation of a customised template, (2) generation of customised prior probability maps, and (3) the main VBM steps: normalisation of the original MR images, segmentation of normalised images, cleaning of grey matter images, modulation of grey matter images, and smoothing of modulated images.

The advantages of this procedure over the traditional simple protocol are that (1) the use of template and prior probability maps computed from the population under study reduces error in the normalisation and segmentation steps, (2) the cleaning step permits removal non-brain voxels erroneously classified as grey matter, ${ }^{17}$ and (3) modulation permits preservation of the original volume of grey matter within each voxel. ${ }^{78} 18$

\section{Customised template}

The customised template was obtained by normalising the images of the 51 MCI patients and 32 controls to the Montreal Neurological Institute template ${ }^{19}$ of SPM99 using a 12 parameter affine transformation, smoothing the normalised images with an $8 \mathrm{~mm}$ isotropic Gaussian kernel and averaging the smoothed images. The anterior commissure was manually set as the origin of the spatial coordinates and images were reoriented coronally perpendicular to the intercommissural line. The normalisation procedure uses a bilinear interpolation algorithm to reslice images to voxel size of $2 \times 2 \times 2 \mathrm{~mm}$. This voxel size is used in the following processing and analysis.

\section{Customised prior probability maps}

Customised prior probability maps were computed by segmenting the normalised images into grey matter (GM), white matter (WM), and cerebrospinal fluid (CSF), then smoothing with an $8 \mathrm{~mm}$ Gaussian filter, and finally averaging the segmented images, thus obtaining the customised prior probability maps specific for GM, WM, and CSF. ${ }^{17}$

\section{Main VBM steps}

Original images were normalised to the customised template through affine and non-linear transformations, medium regularisation, reslicing $2 \times 2 \times 2 \mathrm{~mm}$, and no masking. ${ }^{20}$ The normalised images were segmented into GM, WM, and CSF using the customised prior probability maps. The Xbrain routine, based on erosions and dilatations, was used to remove voxels of non-brain tissue from the segmented images, thus obtaining a brain mask to clean the GM images by intersection with the mask.

In the modulation step, voxel values of the cleaned GM images were multiplied by the measure of relative volumes of warped and unwarped structures derived from the non-linear step of spatial normalisation (Jacobian determinant). ${ }^{17}$ The modulated GM images were smoothed with an $12 \mathrm{~mm}$ isotropic Gaussian kernel.

The final output is a 3D matrix where the three indices are the spatial $x, y$, and $z$ coordinates of voxels in the reference space, and each value of the matrix is proportional to the volume of GM within each voxel. It should be emphasised that the output of each stage of the analysis was visually checked to make sure that the algorithms had actually carried out the expected changes.

\section{Statistical analysis}

A "single subject: conditions and covariates" model was used to compare the volume of GM between MCI and controls on a voxel by voxel basis. The analysis was controlled for apolipoprotein $\mathrm{E}(\mathrm{apoE})$ genotype including genotype $(\epsilon 3 / 3$, $\epsilon 3 / 4 \epsilon$, and $\epsilon 4 / 4)$ as a dummy variable. Intracranial area, age, and sex were included as nuisance covariates. The resulting $t$ map was thresholded at $\mathrm{p}<0.00 \mathrm{l}$, uncorrected. The demographic and clinical data were analysed using one way ANOVA or $\chi^{2}$ test, the level of significant was set at $\mathrm{p}<0.05$.

\section{RESULTS}

There was no difference in age $(\mathrm{F}=1.96, \mathrm{p}=0.17)$, sex $\left(\chi^{2}=0.45, \quad \mathrm{p}=0.50\right)$, education $(\mathrm{F}=0.20, \mathrm{p}=0.66)$, or frequency of the apoE $\epsilon 4$ allele $\left(\chi^{2}=2.5, p=0.11\right)$ between the controls and cases with MCI. As expected, the MiniMental State Examination scores were significantly lower in cases with MCI compared with the controls ( $\mathrm{F}=24.8$, $\mathrm{p}<0.0001$ ) (table 1).

Compared with controls, the cases with MCI had the greatest atrophy in the right hippocampus-amygdala region, and in the right hippocampal tail and thalamus (table 2, fig 1). The medial temporal structures on the left did not quite achieve the level of statistical significance. Other regions with significant GM atrophy were found in the right superior temporal gyrus, the left thalamus, the left inferior temporal gyrus, and the left anterior cingulate gyrus (table 2).

\section{DISCUSSION}

In this study we observed that individuals with MCI have unilateral atrophy in the medial temporal lobe on the right side. Less extensive areas of atrophy were found elsewhere in the temporal lobe, left superior parietal lobule, left cingulate gyrus and, notably, in the thalami bilaterally.

Table 1 Clinical features of the $\mathrm{MCl}^{*}$ cases and controls

\begin{tabular}{llll}
\hline & Controls & MCI & p Value† \\
\hline Number & 32 & 51 & \\
Age (years) & $74(4)$ & $72(5)$ & 0.17 \\
Sex (females, \%) & $19(59 \%)$ & $34(67 \%)$ & 0.66 \\
Education (years) & $7(2)$ & $7(2)$ & 0.67 \\
MMSE score & $27(2)$ & $24(2)$ & $<0.0001$ \\
\hline
\end{tabular}

Results shown as mean (standard deviation).

*Defined by the criteria proposed by Mayo Clinic Alzheimer's Disease Research Center. ${ }^{13}$

†Significance on one way ANOVA or $\chi^{2}$ test.

MMSE, Mini-Mental State Examination. 
Table 2 Atrophic regions of $\mathrm{MCl}$ cases compared with controls ( $p<0.001$, uncorrected)

\begin{tabular}{|c|c|c|c|c|c|}
\hline \multirow[b]{2}{*}{ Cluster size k } & \multirow[b]{2}{*}{ Region } & \multicolumn{3}{|c|}{ Stereotactic coordinates $(\mathrm{mm})$} & \multirow[b]{2}{*}{ Z score } \\
\hline & & $\mathbf{x}$ & $y$ & $\mathbf{z}$ & \\
\hline \multirow[t]{3}{*}{147} & Right thalamus & 12 & -14 & 12 & 3.74 \\
\hline & Right hippocampus (tail) & 24 & -34 & 4 & 3.42 \\
\hline & Right thalamus & 16 & -26 & 0 & 3.31 \\
\hline 246 & Right amygdale-hippocampus & 24 & -4 & -16 & 3.72 \\
\hline 14 & Left superior parietal lobule & -16 & -60 & 66 & 3.36 \\
\hline 10 & Right superior temporal gyrus & 56 & 8 & -6 & 3.31 \\
\hline 6 & Left thalamus & -8 & -14 & 12 & 3.27 \\
\hline 15 & Left anterior cingulate gyrus & -10 & 2 & 42 & 3.24 \\
\hline 2 & Left inferior temporal gyrus & -66 & -20 & -28 & 3.16 \\
\hline 10 & Left thalamus & -16 & -30 & 8 & 3.14 \\
\hline \multicolumn{6}{|c|}{$\begin{array}{l}\text { "Region" denotes the areas of maximal grey matter volume loss within each cluster. For example: the first line } \\
\text { denotes the presence of a } 3 D \text { cluster created from } 147 \text { contiguous voxels of significantly decreased }(p<0.001) \text { grey } \\
\text { matter volume. Within the same cluster there are two more peaks of significance more than } 8 \mathrm{~mm} \text { from the former } \\
\text { and located at } 24,-34,4 \text { and at } 16,-26,0 \text {. } \\
\text { The most significant voxel of the cluster has stereotactic coordinates of } 12,-14 \text {, and } 12 \text { and is located in the region } \\
\text { of the right thalamus. }\end{array}$} \\
\hline
\end{tabular}

To some degree these findings fit comfortably into the widely accepted theory of a hierarchical distribution of $\mathrm{AD}$ pathology - that is, the pathology first starts on the medial temporal lobe, and then spreads to cortical (parietal) association areas. ${ }^{21}$ Our observation, indicating a spread from a predominantly atrophied right medial temporal lobe to the left parietal lobes is in accordance, to some extent, with the so called functional HERA (hemispheric encoding/retrieval asymmetry) model. ${ }^{22}$ Although the HERA model is usually considered to involve mostly the medial temporal lobe and the prefrontal cortices, functional imaging studies have also revealed parietal involvement. ${ }^{23}{ }^{24}$ Thus, as far as the medial temporal lobe and the parietal cortex are considered, these data suggest that MCI does indeed represent preclinical AD. However, we must remember that MCI is a heterogeneous condition and our cohort may include individuals who do not progress to dementia.

To the best of our knowledge, there is only one published study using VBM in MCI, ${ }^{9}$ but with a slightly smaller sample and different operational criteria for MCI. Their data are in
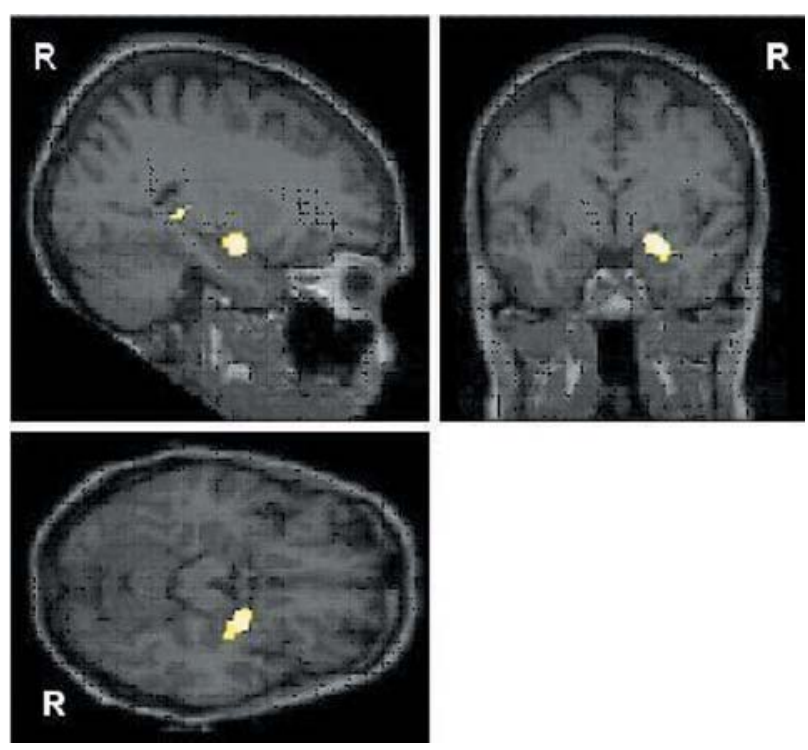

Figure 1 Medial temporal involvement in $\mathrm{MCl}$ patients compared with controls with $p<0.001$, uncorrected. Significant voxels are superimposed on MR images of a single control subject. Atrophy affects the amygdala and the head and tail of the hippocampus. accordance with ours in that they found bilateral thalamic atrophy and some atrophy in the temporal neocortex. Moreover, we found a small area of atrophy in the anterior cingulate cortex. This is of interest because in animal models of $\mathrm{AD}$ it is claimed entorhinal lesions that may be the initial site of $\mathrm{AD}$ pathology, lead to hypometabolism of the cingulate. ${ }^{25}$ In addition, in humans, it should be noted that hypoperfusion of the cingulate has been proposed to predict conversion from MCI to AD. ${ }^{26} 27$ The minor differences between the two studies can be explained by the participants studied, the definition of MCI, and differences in image analysis. However, it would seem that the results between the two studies, in general, are more convergent than divergent.

With respect to the temporal lobe in MCI (and AD), the involvement of regions other than the medial temporal lobe remains a matter of dispute, at least in volumetric studies. ${ }^{45}$ It also should be emphasised that the data obtained by volumetry versus VBM can be highly divergent. ${ }^{17}{ }^{28}$ Thus, a direct comparison between volumetric and VBM studies may be difficult. In fact, this proviso also applies to comparison between different VBM studies. A good example of this is the thalamic involvement found in this study. In our previous VBM study on AD, no thalamic involvement was detected, ${ }^{30}$ nor has it been noted in some other VBM studies on $\mathrm{AD},{ }^{17} 3132$ though others have detected thalamic involvement. ${ }^{20} 2933 \mathrm{~A}$ recent PET study on MCI did hint at thalamic hypoperfusion even when controlled for atrophy seen in MR images. ${ }^{34}$ The pathology of $\mathrm{AD}$ has been suggested to also affect the thalamus, ${ }^{35}$ yet this issue, and the factors underlying the discrepancy, remain to be resolved in future studies. One possibility that is worth mentioning is the bias inherent in the method. As Karas et al stated, the thalamus is one of the structures which "lie geographically in locations which are hard to evaluate with computational models". ${ }^{29}$

Indeed, it should be kept in mind that VBM is a very sensitive and complex method that can be greatly affected by a number of incidental factors. These might explain some discrepancies between our own and other studies such as low quality scans, the reduction of grey/white matter contrast of the elderly or diseased people, and the greater frequency of motion effects in these groups. In addition to these factors, spatial normalisation still produces registration errors with regional specificity: small structures (such as the hippocampus) are highly variable particularly in diseased groups, which might reduce the sensitivity of VBM to detect changes. Methods such as tensor based mapping ${ }^{6}$ might overcome this problem. 


\section{CONCLUSIONS}

In this study we found a unilateral medial temporal atrophy in individuals with MCI. This is a consistent finding in volumetric studies on MCI and invariably detected in volumetric and VBM studies on $\mathrm{AD}$, where the atrophy is bilateral. Some other regions were also found to be atrophic in MCI, but these were relatively small, and it might be premature to draw definitive conclusions based on those findings. However, this study further emphasises the importance of detecting medial temporal atrophy in AD. It also suggests that, as a group, individuals with MCI, defined according to the first version of the Mayo criteria, are more likely to progress to dementia and AD than their age matched peers. Nonetheless, caution must be exercised when interpreting the results due to the heterogeneous nature of MCI. A follow up of the participants of this study is hoped to further clarify this issue.

\section{ACKNOWLEDGEMENTS}

The study was supported by the Research Council for Health of the Academy of Finland, and an EVO grant 5772720 from the Kuopio University Hospital.

\section{Authors' affiliations}

C Pennanen, M P Laakso, M Hallikainen, T Hänninen, M Kivipelto, S Tuomainen, M Vanhanen, H Soininen, Department of Neurology, Kuopio University Hospital, Kuopio, Finland

M P Laakso, M Könönen, R Vanninen, Department of Clinical Radiology, Kuopio University Hospital, Kuopio, Finland C Pennanen, M P Laakso, S Tuomainen, H Soininen, Department of Neuroscience and Neurology, University of Kuopio, Kuopio, Finland E-L Helkala, Department of Public Health, University of Kuopio, Kuopio, Finland

A Nissinen, The National Institute of Public Health, Helsinki, Finland C Testa, M P Laakso, G B Frisoni, The Laboratory of Epidemiology \& Neuroimaging, IRCCS San Giovanni di Dio-FBF, Brescia, Italy G B Frisoni, AFaR- Associazione Fatebenefratelli per la Ricerca, Rome, Italy

C Testa, The Machine Vision Laboratory, Department of Mathematics and Computer Science, University of Udine, Udine, Italy

Competing interests: none declared

\section{REFERENCES}

1 Petersen RC, Stevens JC, Ganguli M, et al. Practice parameter: early detection of dementia: mild cognitive impairment (an evidence-based review). Report of the Quality Standards Subcommittee of the American Academy of Neurology. Neurology 2001;56:1133-42.

2 Ritchie K, Artero S, Touchon J. Classification criteria for mild cognitive impairment: a population-based validation study. Neurology 2001;56:37-42.

3 Larrieu S, Letenneur L, Orgogozo JM, et al. Incidence and outcome of mild cognitive impairment in a population-based prospective cohort. Neurology 2002;59:1594-9.

4 Laakso MP. Structural imaging in cognitive impairment and the dementias: an update. Curr Opin Neurol 2002;15:415-21.

5 Wolf $\mathrm{H}$, Jelic V, Gertz H-J, et al. A critical discussion of the role of neuroimaging in mild cognitive impairment. Acta Neurol Scand 2003; 107(Suppl 179):52-76

6 Ashburner J, Csernansky JG, Davatzikos C, et al. Computer-assisted imaging to assess brain structure in healthy and diseased brains. Lancet Neurol 2003; $2: 79-88$.

7 Ashburner J, Friston KJ. Voxel-based morphometry-the methods. Neuroimage 2000;11:805-21.

8 Good CD, Johnsrude I, Ashburner J, et al. Cerebral asymmetry and the effects of sex and handedness on brain structure: a voxel-based morphometric analysis of 465 normal adult human brains. Neuroimage $2001 ; 14: 685-700$.
9 Chételat G, Desgranges B, de la Sayette $V$, et al. Mapping gray matter loss with voxel-based morphometry in mild cognitive impairment. NeuroReport 2002;13:1939-43.

10 Kivipelto $M$, Helkala E-L, Hänninen T, et al. Midlife vascular risk factors and late-life mild cognitive impairment. A population-based study. Neurology 2001;56:1683-89.

11 Hänninen T, Hallikainen M, Tuomainen S, et al. Prevalence of mild cognitive impairment: a population-based study in elderly subjects. Acta Neurol Scand 2002; 106: 148-54

12 Pennanen C, Kivipelto M, Tuomainen S, et al. Hippocampus and entorhinal cortex in mild cognitive impairment. Neurobiol Aging 2004;25:303-10.

13 Petersen RC, Smith GE, Ivnik RJ, et al. Apolipoprotein E status as a predictor of the development of Alzheimer's disease in memory-impaired individuals. JAMA 1995;273:1274-8.

14 Smith GE, Petersen RC, Parisi JE, et al. Definition, course, and outcome of mild cognitive impairment. Aging Neuropsychol Cogn 1996;3:141-7.

15 Tsukamoto K, Watanabe T, Matsushima T, et al. Determination by PCR-RFLP of apo E genotype in a Japanese population. J Lab Clin Med 1993; 121:598-602.

16 Rorden C, Brett M. Stereotaxic display of brain lesions. Behav Neurol 2001:112:191-200.

17 Good CD, Scahill RI, Fox NC, et al. Automatic differentiation of anatomical patterns in the human brain: validation with studies of degenerative dementias. Neurolmage 2002;17:29-46.

18 Ashburner J, Friston KJ. Why voxel-based morphometry should be used. Neurolmage $2001 ; 14: 1238-43$.

19 Evans AC, Kamber M, Collins DL, et al. An MRI-based probabilistic atlas of neuroanatomy. In: Shorvon S, Fish D, Andermann F, et al, eds. Magnetic resonance scanning and epilepsy. New York: Plenum Press, 1994:263-74.

20 Baron JC, Chételat $G$, Desgranges $B$, et al. In vivo mapping of gray matter loss with voxel-based morphometry in mild Alzheimer's disease. Neurolmage 2001;14:298-309.

21 Braak H, Braak E. Neuropathological stageing of Alzheimer-related changes. Acta Neuropathol 1991;82:239-59.

22 Habib R, Nyberg L, Tulving E. Hemispheric asymmetries of memory: the HERA model revisited. Trends Cogn Sci 2003;7:241-5.

23 Cabeza R, Grady CL, Nyberg L, et al. Age-related differences in neural activity during memory encoding and retrieval: a positron emission tomography study. J Neurosci 1997; 17:391-400.

24 Desgranges B, Baron JC, Eustache F. The functional neuroanatomy of episodic memory: the role of the frontal lobes, the hippocampal formation, and other areas. Neurolmage 1998;8:198-213.

25 Meguro K, Blaizot X, Kondoh Y, et al. Neocortical and hippocampal glucose hypometabolism following neurotoxic lesions of the entorhinal and perirhinal cortices in the non-human primate as shown by PET. Implications for Alzheimer's disease. Brain 1999;122:1519-31.

26 Huang C, Wahlund L-O, Svensson L, et al. Cingulate cortex hypoperfusion predicts Alzheimer's disease in mild cognitive impairment. BMC Neurol 2002;2:9.

27 Okamura N, Arai H, Maruyama M, et al. Combined analysis of CSF tau levels and $\left[{ }^{123}\right]$ iodoamphetamine SPECT in mild cognitive impairment: implications for a novel predictor of Alzheimer's disease. Am J Psychiatry 2002;159:474-6.

28 Tisserand DJ, Pruessner JC, Sanz Arigita EJ, et al. Regional frontal cortical volumes decrease differentially in aging: an MRI study to compare volumetric approaches and voxel-based morphometry. Neurolmage 2002; 17:657-69.

29 Karas GB, Burton EJ, Rombouts SARB, et al. A comprehensive study of gray matter loss in patients with Alzheimer's disease using optimized voxel-based morphometry. Neurolmage 2003;18:895-907.

30 Frisoni GB, Testa C, Zorzan A, et al. Detection of grey matter loss in mild Alzheimer's disease with voxel based morphometry. J Neurol Neurosurg Psychiatry 2002;73:657-64.

31 Ohnishi T, Matsuda H, Tabira T, et al. Changes in brain morphology in Alzheimer disease and normal aging: is Alzheimer disease an exaggerated aging process? Am J Neuroradiol 2001;22:1680-5.

32 Busatto GF, Garrido GEJ, Almeida OP, et al. A voxel-based morphometry study of temporal lobe gray matter reductions in Alzheimer's disease. Neurobiol Aging 2003:24:221-31.

33 Burton EJ, Karas G, Paling SM, et al. Patterns of cerebral atrophy in dementia with Lewy bodies using voxel-based morphometry. Neurolmage 2002; 17:618-30.

34 Nestor PJ, Fryer TD, Smielewski P, et al. Limbic hypometabolism in Alzheimer's disease and mild cognitive impairment. Ann Neurol 2003;54:343-51

35 Braak H, Braak E. Alzheimer's disease affects limbic nuclei of the thalamus Acta Neuropathol 1991;81:261-8. 